\title{
White Dwarf Central Stars of Planetary Nebulae
}

\author{
Ralf Napiwotzki \\ Dr. Remeis-Sternwarte, Sternwartstr. 7, 96049 Bamberg, Germany
}

\begin{abstract}
Results of a spectroscopic investigation of central stars of old planetary nebulae (PNe) are reported. The evolutionary status of the central stars is discussed and it is shown that most are in good agreement with standard post-AGB evolution, but some are best explained as descendents from the first RGB after binary interaction. The distance scale of $\mathrm{PNe}$ is discussed.
\end{abstract}

\section{Introduction}

Central stars of planetary nebulae (CSPNe) are the immediate precursors of white dwarfs. Two distinct sequences of CSPNe and white dwarfs are known: a hydrogen-rich and a hydrogen-deficient (helium- and carbon-rich) one. Fontaine \& Wesemael (1987) discussed a lack of very hot white dwarfs in the hydrogen-rich sequence, which contradicts the predictions of the canonical post-AGB evolutionary calculations. This prompted us to start a spectroscopic survey of the nuclei of old PNe. These CSPNe are far evolved, just entering the white dwarf cooling track, and therefore ideal objects to test the early evolution of white dwarfs. We selected apparently very old, faint and extended nebulae whose central stars could be identified. Low and medium resolution spectra were obtained with the TWIN spectrograph at the $3.5 \mathrm{~m}$ telescope of the Calar Alto observatory. The observations and the data reduction procedures are described in Napiwotzki \& Schönberner (1995) and Napiwotzki (1999).

\section{Analysis and evolutionary status}

Our sample contained a total of 39 CSPNe. It turned out that only seven stars are hydrogen-poor while 28 central stars are hydrogen-rich (cf. Napiwotzki \& Schönberner, 1995, for individual classifications). For the determination of the precise evolutionary status of the CSPNe the knowledge of the fundamental parameters temperature and surface gravity is crucial. Therefore, we performed a model atmosphere analysis of the hydrogen-rich objects. We calculated a grid of NLTE model atmospheres composed of hydrogen and helium with the NLTE code developed by Werner (1986). We followed the standard procedure for

\footnotetext{
${ }^{6}$ Visiting astronomer, German-Spanish Astronomical Center, Calar Alto, Spain, operated by the Max-Planck-Institut für Astronomie, Heidelberg, jointly with the Spanish National Commission for Astronomy
} 


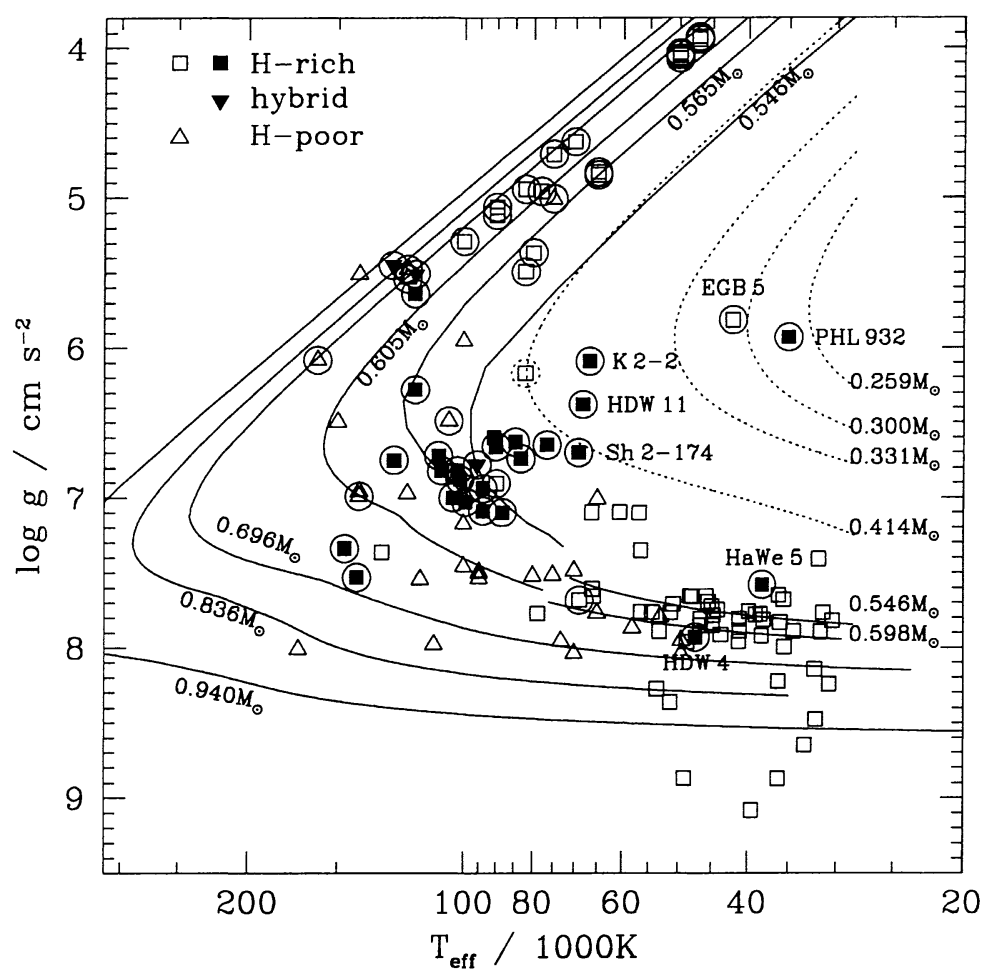

Figure 1. Temperature-gravity diagram with the results of the new analyses plotted as filled symbols and literature values as open symbols (cf. Napiwotzki 1999 for references). CSPNe are encircled. Post-AGB evolutionary tracks (Blöcker et al. 1995; Schönberner 1993; Koester \& Schönberner 1986) are drawn as solid lines, labelled with the remnant mass, the post-RGB tracks of Driebe et al. (1998) as dashed lines.

the analysis of hydrogen-rich DA white dwarfs. Both, temperature and surface gravity, are determined from a simultaneous fit of the Balmer lines. Although this method works very well for white dwarfs it failed for nearly all hydrogenrich central stars of old PNe (Napiwotzki \& Rauch 1994; Napiwotzki 1999): no consistent fit of all Balmer lines was possible. A strong trend is present: fitting of higher Balmer lines yielded higher temperatures. However, theoretical and empirical considerations (Napiwotzki 1993; Werner 1996 and these proceedings) provide evidence that the temperatures of the higher Balmer lines are essentially correct. Hence we adopted the temperatures from the highest available Balmer line only (details given by Napiwotzki 1999).

The position of the stars in the temperature-gravity plane is shown in Fig. 1. Our new analyses of central stars of old PNe fill nicely the reported "gap" in the hydrogen-rich sequence. Therefore evolutionary scenarios claiming a hydrogenpoor stage of all (or most) pre-white dwarfs (Fontaine \& Wesemael 1987) can be ruled out.

The position of most CSPNe in Fig. 1 is well explained by the canonical post-AGB evolutionary tracks. A subset (K 2-2, HDW 11, Sh 2-174, EGB 5, 

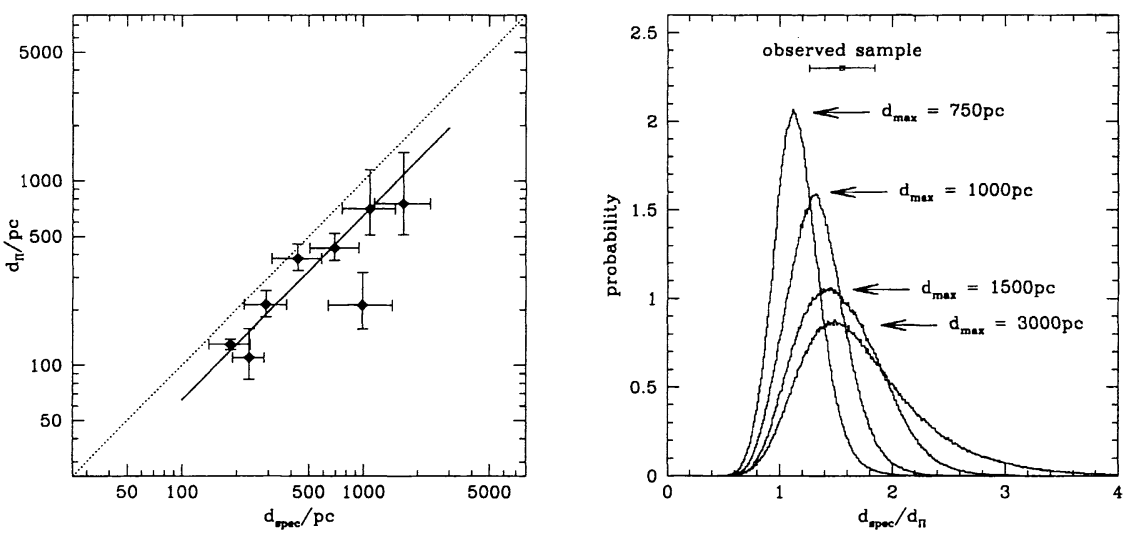

Figure 2. Left: Distances computed from the results of the spectral analysis compared to trigonometric distances. Right: Results of the Monte Carlo simulation.

and PHL 932) lie in a region apart from post-AGB tracks. Their fundamental parameters are in agreement with tracks of post-RGB stars, whose hydrogen-rich envelope was stripped away by a companion during the (first) red giant branch phase. The remaining mass is below the limit for the ignition of helium burning and the star will eventually become a low mass white dwarf with a helium core ("post-RGB evolution"). It is commonly assumed that post-RGB evolution is too slow to produce a PN. However, a closer examination shows that for Sh 2174 and K 2-2 the kinematical ages of the PNe (40000 years and 65000 years, respectively) are are in very good agreement with the evolutionary ages predicted by the computations ( 100000 years for both), if the given uncertainties of both age estimates are taken into account. The agreement is worse for HDW 11, PHL 932, and EGB 5. We conclude that the cases of Sh 2-174 and K 2-2 strongly argue that post-RGB stars can form PNe during their evolution to low mass white dwarfs.

\section{Distance scales}

Our sample of white dwarf CSPN is large enough for a statistically meaningful comparison with other distance scales. Accurate trigonometric parallaxes have been measured for eight CSPNe of our sample from the ground (Harris et al. 1997 ) or by HIPPARCOS (Pottasch \& Acker 1998). The resulting distance values $d_{\Pi}$ are compared to our spectroscopic results in Fig. 2. The trigonometric distances are always smaller. The weighted mean of the distance ratios $d_{\text {spec }} / d_{\Pi}$ amounts to $1.55 \pm 0.29$. Taken at face value this would indicate that the model atmosphere results overestimate the distances. However, one must consider that trigonometric distances are subject to heavy biases, which are introduced by random errors of measurement and on average cause the parallaxes to be overestimated.

We performed a Monte Carlo simulation to derive biases caused by the selection of CSPNe for parallax measurements and accompanying measurement 
errors. Our simulation created a simple model of the Galactic CSPNe population and applied selection criteria and measurement errors similar to the existing sample (details are given in Napiwotzki 2001). The working hypothesis for the simulations was that the spectroscopic distance scale does not deviate from the correct distances. Nevertheless, we found that the "measured" ratio $d_{\text {spec }} / d_{\Pi}$ of the simulated samples is always considerably larger than unity. The exact value depends (besides the measurement accuracy) sensitively on the pre-selection criterium for parallax measurements. This effect is demonstrated in Fig. 2. Usually parallaxes are measured for CSPN, which are known (e.g. from spectroscopic analysis) to have a distance below a certain limit $d_{\max }$. Judged from our analysis the value of $d_{\max }$ for the current sample of CSPNe with trigonometric parallaxes lies in the range between $1000 \mathrm{pc}$ and $1500 \mathrm{pc}$. This corresponds to $d_{\text {spec }} / d_{\Pi}=1.32 \pm 0.25$ and $1.50 \pm 0.38$, respectively. Fig. 2 demonstrates that the mean value of $d_{\text {spec }} / d_{\Pi}$ of our observed sample is well within the range predicted by Monte Carlo simulations for $d_{\max }=1000 \ldots 1500$ and perfect agreement of both distance scales. Thus the trigonometric parallax measurements provide no evidence that the spectroscopic distances are in error, but confirm the spectroscopic distance scale, instead. A more stringent test can be expected from a larger sample with better defined limits $d_{\max }$.

A standard tool for PN research is the determination of "statistical" distances by Shklovsky type methods. A comparison of the spectroscopic distances of the white dwarf CSPNe with distance determinations of the Shklovsky type revealed large discrepancies. We derive a mean ratio $d_{\text {spec }} / d_{\text {Shkl }}=3.2 \pm 1.3$ (peculiar objects not in agreement with canonical post-AGB evolution excluded). This indicates disagreement of both distance scales on a level far beyond any uncertainty discussed above. A strong trend with the PN radii is present: Shklovsky distances of young, small PNe are too large, while they are too small for large evolved PNe. We conclude that the Shklovsky method (and other related procedures) can only be used to produce very rough distance estimates.

\section{References}

Blöcker, T. 1995 A\&A 299, 755

Driebe, T., Schönberner, D., Blöcker, T., \& Herwig, F. 1998, A\&A 339, 123

Fontaine, G., \& Wesemael, F. 1987, in IAU Coll. 95, eds. A.G.D. Philip, D.S. Hayes, J.W. Liebert (L. Davis Press), 319

Harris, H.C., Dahn, C.C., Monet, D.G., \& Pier, J.R. 1997, in IAUS 180, Planetary Nebulae, ed. H.J. Habing \& H.J.G.L.M. Lamers (Kluwer, Dordrecht), 40

Koester, D., \& Schönberner, D. 1986, A\&A 154, 125

Napiwotzki, R. 1993, Acta Astron. 43, 343

Napiwotzki, R. 1999, A\&A 350, 101

Napiwotzki, R. 2001, A\&A 367, 973

Napiwotzki, R., \& Schönberner, D. 1995, A\&A 301, 545

Pottasch, S.R. \& Acker, A. 1998, A\&A 329, L5

Schönberner, D. 1983, ApJ 272, 708

Werner, K. 1986, A\&A 161, 177

Werner, K. 1996, ApJ 457, L39 